\title{
Differentiation of adipose-derived stem cells into functional chondrocytes by a small molecule that induces Sox9
}

Jiyun Lee ${ }^{1,2}$, Chang Youn Lee ${ }^{3}$, Jun-Hee Park ${ }^{3}$, Hyang-Hee Seo ${ }^{1}$, Sunhye Shin ${ }^{3}$, Byeong-Wook Song $\mathbb{D}^{2}$, II-Kwon Kim², Sang Woo Kim², Seahyoung Lee ${ }^{2}$, Jong-Chul Park ${ }^{1,4}$, Soyeon Lim $\mathbb{B}^{2}$ and Ki-Chul Hwang $\mathbb{B}^{2}$

\begin{abstract}
Osteoarthritis $(\mathrm{OA})$ is a common joint disease that results from the disintegration of joint cartilage and the underlying bone. Because cartilage and chondrocytes lack the ability to self-regenerate, efforts have been made to utilize stem cells to treat OA. Although various methods have been used to differentiate stem cells into functional chondrocytes, the currently available methods cannot induce stem cells to undergo differentiation into chondrocyte-like cells without inducing characteristics of hypertrophic chondrocytes, which finally lead to cartilage disintegration and calcification. Therefore, an optimized method to differentiate stem cells into chondrocytes that do not display undesired phenotypes is needed. This study focused on differentiating adipose-derived stem cells (ASCs) into functional chondrocytes using a small molecule that regulated the expression of Sox9 as a key factor in cartilage development and then explored its ability to treat OA. We selected ellipticine (ELPC), which induces chondrocyte differentiation of ASCs, using a GFP-Sox9 promoter vector screening system. An in vivo study was performed to confirm the recovery rate of cartilage regeneration with ASC differentiation into chondrocytes by ELPC in a collagenase-induced animal model of OA. Taken together, these data indicate that ellipticine induces ASCs to differentiate into mature chondrocytes without hypertrophic chondrocytes in vitro and in vivo, thus overcoming a problem encountered in previous studies. These results indicate that ELPC is a novel chondrocyte differentiationinducing drug that shows potential as a cell therapy for OA.
\end{abstract}

\section{Introduction}

Osteoarthritis (OA) is a major degenerative and common chronic joint disease caused mainly by aging ${ }^{1,2}$. Moreover, OA has been observed in younger populations due to systemic and genetic factors ${ }^{3-5}$. The articular cartilage composed of chondrocytes and a dense extracellular matrix (ECM) is a key component of joints that have a limited capacity for intrinsic healing and repair ${ }^{6,7}$. During the progression of OA, active ECM remodeling

\footnotetext{
Correspondence: Soyeon Lim (slim724@cku.ac.kr) or Ki-

Chul Hwang (kchwang@cku.ac.kr)

'Brain Korea 21 PLUS Project for Medical Science, Yonsei University, Seoul, Republic of Korea

${ }^{2}$ Institute for Bio-Medical Convergence, College of Medicine, Catholic Kwandong University, Gangneung, Republic of Korea

Full list of author information is available at the end of the article.
}

occurs with environmental changes such as severe or chronic inflammation, which in turn leads to the induction of the maturation of hypertrophic chondrocytes. Hypertrophic chondrocytes characteristically overexpress hypertrophic markers, runt-related transcription factor 2 (RUNX2), Type X collagen, Indian hedgehog, and Transglutaminase-2. Hypertrophic chondrocytes are also known to synthesize various matrix-degrading enzymes, such as matrix metalloproteinase 13 (MMP13) and a disintegrin and metalloproteinase containing thrombospondin motifs (ADAMTS4, 5), which accelerate the decomposition of the ECM, leading to cartilage loss and calcification $^{8,9}$

Although various methods from alternative medicine to surgical procedures have been utilized for OA patients, 
there is no way to reverse the onset of this disease. The most recent therapeutic approaches for OA involve the use of stem cells ${ }^{10}$. Adipose-derived stem cells (ASCs) have recently been widely used because they are easier to obtain than bone marrow-derived stem cells ${ }^{11,12}$. However, previous studies have demonstrated that hypertrophic chondrocytes are a major problem in differentiated chondrocytes using stem cells ${ }^{13,14}$.

To regulate the expression of critical genes and proteins involved in stem cell differentiation, previous studies have used systems such as viral vectors, microRNAs, siRNAs, and shRNAs. However, conventional systems have limitations regarding cytotoxicity, delivery, and maintenance ${ }^{15-17}$. Therefore, we used small molecules that are stable and transferable in the cells to control the expression of genes and proteins. Consequently, a new small molecule-based method was used to screen for small molecule drugs that could induce the differentiation from ASCs into chondrocytes. Ultimately, the goal is to induce the optimal differentiation of ASCs into mature chondrocytes using a new small molecule-based method.

\section{Materials and methods \\ Materials}

Ellipticine (ELPC, 5,11-dimethyl-6H-pyrido[4,3-b]carbazole) was purchased from Enzo Life Science, Inc. In-house chemical libraries, receptor agonists and antagonist kinase inhibitors, and ion channel activators and inhibitors were used ${ }^{18}$. Dulbecco's modified Eagle's medium (DMEM), fetal bovine serum (FBS), and penicillin-streptomycin were obtained from the same corporation (Life Technologies, Grand Island, NY, USA). For polymerase chain reaction (PCR), oligonucleotides were synthesized by Bioneer (Bioneer, Daejeon, South Korea), and RNA was extracted using chloroform and 2propanol (Sigma-Aldrich, St. Louis, MO, USA). Reverse transcription for cDNA synthesis was conducted using a reverse transcription system (Promega Corporation, Madison, WI, USA), and PCR was performed with Ex Taq, dNTP mixture (2.5 mM each), and 10x Ex Taq buffer (TaKaRa Bio, Inc., Otsu, Japan). Western blot and immunofluorescence antibodies were used to detect type II collagen, aggrecan, type X collagen, p53, p-p53, and $\beta$-actin (Santa Cruz Biotechnology, USA). Secondary antibody usage was divided into 2 types: mouse or rabbit (Enzo Life Sciences, Inc., Farmingdale, NY, USA) for western blot analysis (horseradish peroxidase (HRP)conjugated) and mouse, rabbit, or goat (Jackson Immunoresearch Laboratories, West Grove, PA, USA; Vector Laboratories, Burlingame, CA, USA; Abcam, USA) for immunostaining (fluorescein isothiocyanate, FITC; phycoerythrin, PE; or allophycocyanin, APC-conjugated). Western blotting detection systems were obtained from
GE Healthcare Life Sciences (Uppsala, Sweden), and 4',6diamidino-2-phenylindole was obtained from Life Technologies (USA).

\section{Sox9 promoter gene assay}

The promoter region for the sex-determining region Ytype high mobility group box (Sox) 9 (-1034 bp to $+67 \mathrm{bp}$ ) used in this study was based on the promoter region described in DC Colter et al. ${ }^{19}$. The PCR product of the promoter region was cloned into the pAcGFP1-1 vector. The conjugated Sox9 promoter GFP vector was transfected into ASCs using the TransIT-X2 Dynamic Delivery system. After transfection, the GFP intensity was examined under fluorescence microscopy $\left(\right.$ Glomax $^{\circledR}$ Explorer multimode microplate reader, Promega, WI, USA).

\section{Animals}

Adult 8-week-old Sprague-Dawley male rats were used (Koatech, Pyeongtaek, Korea) from Harlan USA. All animal experimental procedures were approved by the Institutional Animal Care and Use Committee, Catholic Kwandong University College of Medicine, and the Association for Assessment and Accreditation of Laboratory Animal Care and were performed in accordance with the Guidelines and Regulations for Animal Care.

\section{Adipose-derived stem cell culture}

Human ASCs were purchased from Invitrogen (CA, USA) and cultured in growth media (DMEM supplemented with $10 \% \mathrm{FBS}, 100 \mathrm{unit} / \mathrm{ml}$ penicillin, and $100 \mu \mathrm{g} / \mathrm{ml}$ streptomycin) at $37^{\circ} \mathrm{C}$ and $5 \% \mathrm{CO}_{2}$. Experiments used cells between passages 3 and 7 for differentiation.

\section{Pellet culture system}

ASCs were cultured by an improved pellet culture system described by Johnstone et $\mathrm{al}^{20}$ When cartilage formation proceeds in a stable and smooth manner, the pellet will maintain a rounded shape while it grows. ASCs $\left(2.5 \times 10^{5}\right)$ were centrifuged at $600 \times g$ for $5 \mathrm{~min}$ in a $15-\mathrm{ml}$ polypropylene tube. The resulting pellets were treated with an ELPC final concentration of $1 \mu \mathrm{M}$ in $10 \% \mathrm{FBS}$ DMEM for 16 days. The medium and the ELPC were replaced with fresh medium and ELPC once every 3 days.

\section{Cell viability assay}

ASCs were plated in 96-well cell culture plates in triplicate at $5 \times 10^{3}$ cells/well. After treatment with ELPC for $24 \mathrm{~h}$ or $48 \mathrm{~h}$ in ASCs, EZ-Cytox reagent (DoGEN, Seoul, Korea) was added to each well and incubated at $37^{\circ} \mathrm{C}$ for $2 \mathrm{~h}$ to react. The sample absorbance was measured using a microplate reader (Thermo Fisher Scientific, MA, USA) at $450 \mathrm{~nm}$. 
Reverse transcription-polymerase chain reaction (RT-PCR)

Total RNA was extracted using TRIzol. Chloroform was added to separate each sample into layers of RNA, DNA, and proteins, and then, each sample was centrifuged at $12,000 \mathrm{rpm}$ and $4{ }^{\circ} \mathrm{C}$ for $15 \mathrm{~min}$. Next, the RNA from each sample was collected in a new tube, and 2-propanol was added to obtain the pellet, after which the centrifugation was repeated at $12,000 \mathrm{rpm}$ at $4{ }^{\circ} \mathrm{C}$ for $10 \mathrm{~min}$. The pellet was washed in $75 \%(\mathrm{v} / \mathrm{v})$ ethanol and mixed with diethylpyrocarbonate (DEPC) dissolved in water. After centrifugation at approximately $12000 \mathrm{rpm}$ at $4{ }^{\circ} \mathrm{C}$ for $5 \mathrm{~min}$, the pellet was dried at room temperature. Finally, the pellet was dissolved in $30 \mu \mathrm{L}$ of nuclease-free water (NFW). The quality and quantity of RNA were estimated by calculation of OD260/OD280 ratios using a spectrophotometer. Complementary DNA (cDNA) was synthesized using the RT System kit. The RNA was added to the oligo dT primer, dNTP mixture, RTase, RNase inhibitor, and buffer. The generated cDNA was mixed with each primer, dNTP mixture, Taq polymerase, and reaction buffer in the PCR tube. PCR conditions consisted of denaturation at $94^{\circ} \mathrm{C}$ for $3 \mathrm{~min}$, followed by 30 cycles each featuring denaturation at $94{ }^{\circ} \mathrm{C}$ for $30 \mathrm{~s}$, annealing at $48-60^{\circ} \mathrm{C}$ for $30 \mathrm{~s}$, and elongation at $72{ }^{\circ} \mathrm{C}$ for $30 \mathrm{~s}$, and then, the reaction was maintained at $72^{\circ} \mathrm{C}$ for $10 \mathrm{~min}$. The following primer sequences were used: F: GAGGAAG TCGGTGAAGAACG and R: ATCGAAGGTCTCGATG TTGG for Sox9; F: TGAGGAGGGCTGGAACAAGT and R: GGAGGTGGTAATTGCAGGGA for Aggrecan; F: TG GAGAAACCATCAATGGTGG and R: TGGAGAAACC ATCAATGGTGG for Type II collagen; F: ATGACCCAA GGACTGGAATCTTTA and R: ATGACCCAAGGACT GGAATCTTTA for Type X collagen; F: AAGGGTCCAC TCTGGCTTTG and R: CTAGGCGCATTTCAGGTGCT for RUNX2; F: TTTCCCTGGCAAGGACTATG and R: GGAGGAGAACTGGACACCAC for ADAMTS4; F: TG ACCATGAGGAGCACTACG and R: TGGGAGAGGCC AAGTAAATG for ADAMTS5; F: GTGGTGTGGGAAG TATCATCA and R: GCATCTGGAGTAACCGTATTG for MMP13; F: GAAACTACTTCCTGAAAACAACGT and R: GCCTCACAACCTCCGTACT for p53; F: CATG GGTGTGAACCATGAGA and R: GGTCATGAGTCCT TCCACGA for GAPDH. PCR products were separated by electrophoresis on $1.2 \%(\mathrm{w} / \mathrm{v})$ agarose gels. Gel-Doc was used to visualize the bands.

\section{Nuclear extraction}

The nuclei and cytoplasm of ASCs were extracted using NE-PER nuclear and cytoplasmic extraction reagents kits (Thermo Scientific, IL, USA). ASCs were harvested with trypsin-EDTA and centrifuged at $500 \times g$ for $5 \mathrm{~min}$ to collect the cell pellet. Then, the cell pellet was washed with PBS, and $1-10 \times 10^{6}$ cells were transferred to a 1.5 $\mathrm{ml}$ microcentrifuge tube and pelleted by centrifugation at
$500 \times g$ for $3 \mathrm{~min}$. After the PBS was removed, cytoplasmic extraction reagent I (CER I) was added to the cell pellet. The cell pellet was vortexed to fully suspend it on the highest setting for $15 \mathrm{~s}$ and then incubated on ice for $10 \mathrm{~min}$. Then, cytoplasmic extraction reagent II (CER II) was added to the sample, vortexed for $5 \mathrm{~s}$, placed on ice for $1 \mathrm{~min}$, vortexed for $5 \mathrm{~s}$, and centrifuged for $5 \mathrm{~min}$ at maximum speed in a microcentrifuge $(\sim 16,000 \times g)$. The supernatant was immediately transferred to a new tube and stored on ice until use. The supernatant contained cytoplasmic proteins, and the pellet contained the nuclear fraction. After addition of ice-cold nuclear extraction reagent (NER) to the pellet, vortexing for $15 \mathrm{~s}$ and incubation on ice for $10 \mathrm{~min}$ was repeated four times. Then, the sample was centrifuged at maximum speed in a microcentrifuge $(\sim 16,000 \times g)$ for $10 \mathrm{~min}$, and the supernatant fraction was transferred to a new tube. The supernatant included the nuclear extract.

\section{Western blot analysis}

The cells were washed with PBS and lysed in lysis buffer with proteinase and phosphatase inhibitors. Protein concentrations were determined using the BCA Protein Assay Kit. Then, the same concentration of proteins was separated on a sodium dodecyl sulfate-polyacrylamide gel and transferred to a polyvinylidene difluoride membrane. After the membrane was blocked in 5\% skim milk mixed with $0.1 \%$ Tween 20 in TBS buffer for $1 \mathrm{~h}$ at room temperature, it was rinsed twice with TBS buffer and incubated with primary antibody overnight at $4{ }^{\circ} \mathrm{C}$. Next, the membrane was washed three times with $0.1 \%$ Tween 20 TBS buffer for $10 \mathrm{~min}$ and incubated for $1 \mathrm{~h}$ at room temperature with HRP-conjugated secondary antibodies. After extensive washing, the bands were detected with an enhanced chemiluminescence reagent. The band intensities were quantified using $\mathrm{NIH}$ ImageJ version $1.34 \mathrm{e}$ software.

\section{ADAMTS4 and ADAMTS5 ELISA verification}

The protein activities of ADAMTS4 and ADAMTS5, which are released as active protease forms in the cell culture supernatant, were measured using ADAMTS4 and ADAMTS5 ELISA kits (R\&D Systems). The ADAMTS4 and ADAMTS5 capture antibodies were coated in a 96-well microplate overnight at room temperature. After three washes, blocking with reagent diluent was performed for $1 \mathrm{~h}$, and the standard and sample were added and reacted at room temperature for $2 \mathrm{~h}$. The plate was washed three times, incubated with detection antibody for $2 \mathrm{~h}$, and washed again. Streptavidin-HRP was added and reacted for $20 \mathrm{~min}$ in the dark. After the substrate solution was added for $20 \mathrm{~min}$, the reaction was stopped using stop solution, and the value was read at $450 \mathrm{~nm}$ using a microplate reader. 


\section{Transcription factor activation array}

Transcription factor activation was measured using a TF activation profiling plate array and customized array (Signosis, Inc., CA, USA). The nuclei of ASCs were extracted using a Nuclear Extraction Kit (Signosis, Inc.). ASCs were washed with PBS, and then, the Buffer I working reagent mixture was added for $10 \mathrm{~min}$. The ASCs were removed from the plate using a scraper, transferred to a microcentrifuge tube and centrifuged at $12000 \mathrm{rpm}$ for $5 \mathrm{~min}$. The supernatant was discarded completely, and the Buffer II working reagent mixture was added for $2 \mathrm{~h}$. The sample was centrifuged at 12,000 rpm for $5 \mathrm{~min}$, and then, the supernatant was transferred to a new tube. For TF/DNA complex formation, the TF probe and nuclear fraction were mixed in a tube for $30 \mathrm{~min}$. The TF/DNA complex was separated from the free probe and incubated in a hybridization plate overnight. Finally, transcription factor activity was detected using a luminometer.

\section{Osteoarthritis induction and ASC injection}

Osteoarthritis was induced using $250 \mathrm{~g}$ male SpragueDawley (SD) rats. Rats were anesthetized by intraperitoneal administration of $20 \mathrm{mg} / \mathrm{kg}$ Zoletil and $5 \mathrm{mg} / \mathrm{kg}$ Rompun. Anesthesia was performed twice by intraarticular injection for 1 week, where $30 \mu \mathrm{l}$ of either sterile saline (control group) and $250 \mathrm{U}$ or $500 \mathrm{U}$ of type II collagenase dissolved in saline and filtered through a $0.22-$ $\mu \mathrm{m}$ membrane was injected. ASCs induce differentiation into chondrocytes after treatment with ELPC for 16 days prior to injection. One week after the injection of Type II collagenase, normal ASCs and differentiated ASCs were injected with $1 \times 10^{6}$ cells in PBS.

\section{Alcian blue staining}

Alcian blue is a blue stain associated with the sulfated glycosaminoglycan of the cartilage matrix. After 16 days, the cells were rinsed once with PBS and fixed with $4 \%$

\section{a}
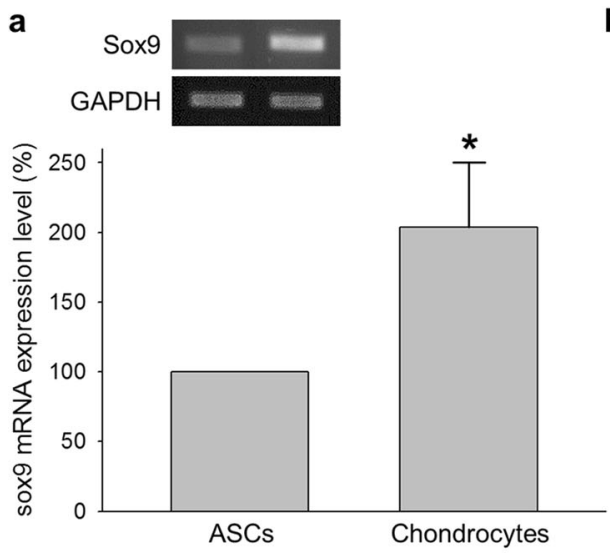

C

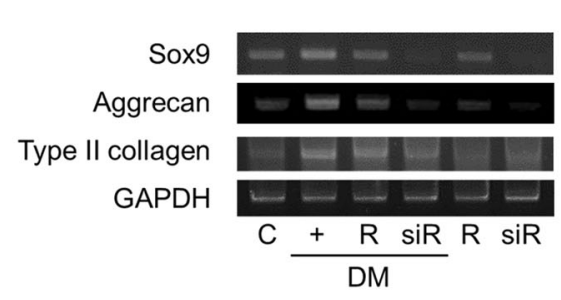

b
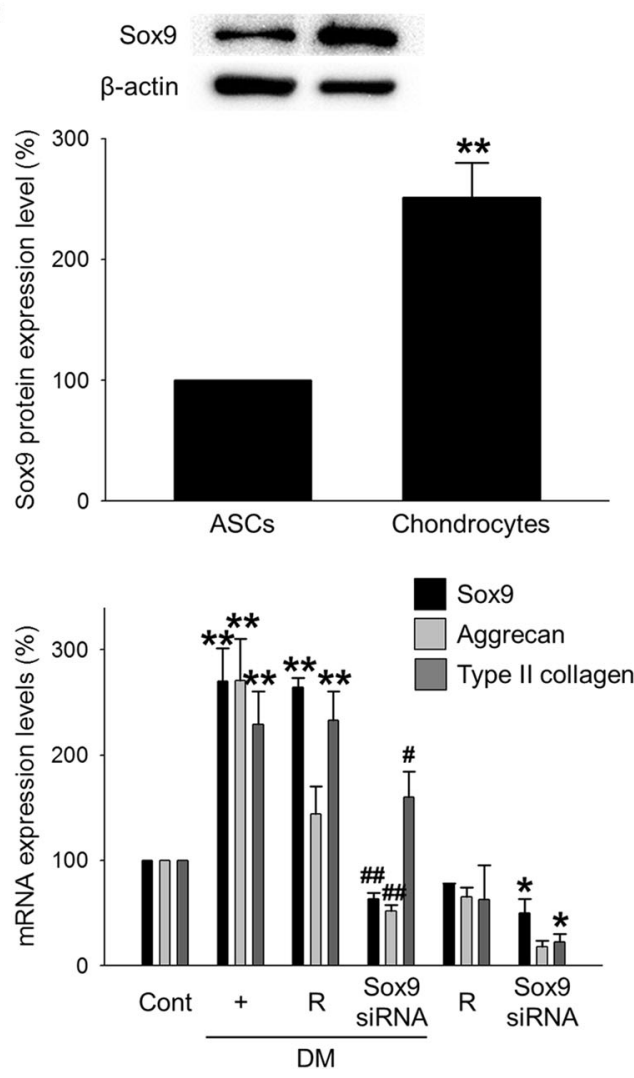

Fig. 1 Importance of Sox 9 in chondrogenic differentiation. $\mathbf{a}, \mathbf{b}$ mRNA and protein expression of Sox 9 in ASCs and chondrocytes. Normalized by GAPDH and B-actin. ${ }^{*} p<0.05$ compared to ASCs ${ }^{* *} p<0.001$ compared to ASCs. $n=3$. c ASCs were transfected with $50 \mathrm{nM}$ Sox9 siRNA for $24 \mathrm{~h}$ and cultured in chondrocyte differentiation medium (DM). The cells were transfected with the Sox9 siRNA every 3 days for 16 days while cultured in DM. The mRNA expression of Sox9, Aggrecan, and Type II collagen in the Sox9 siRNA-transfected ASCs. C and Cont control, R reagent-transfected ASCs, siR Sox9 siRNA-transfected ASCS, DM chondrogenic differentiation induction medium. ${ }^{*} p<0.05,{ }^{* *} p<0.001$ compared to the control, ${ }^{\#} p<0.05,{ }^{\# \#} p<$ 0.001 compared to the reagent-transfected and DM-treated ASCs. $n=3$. 


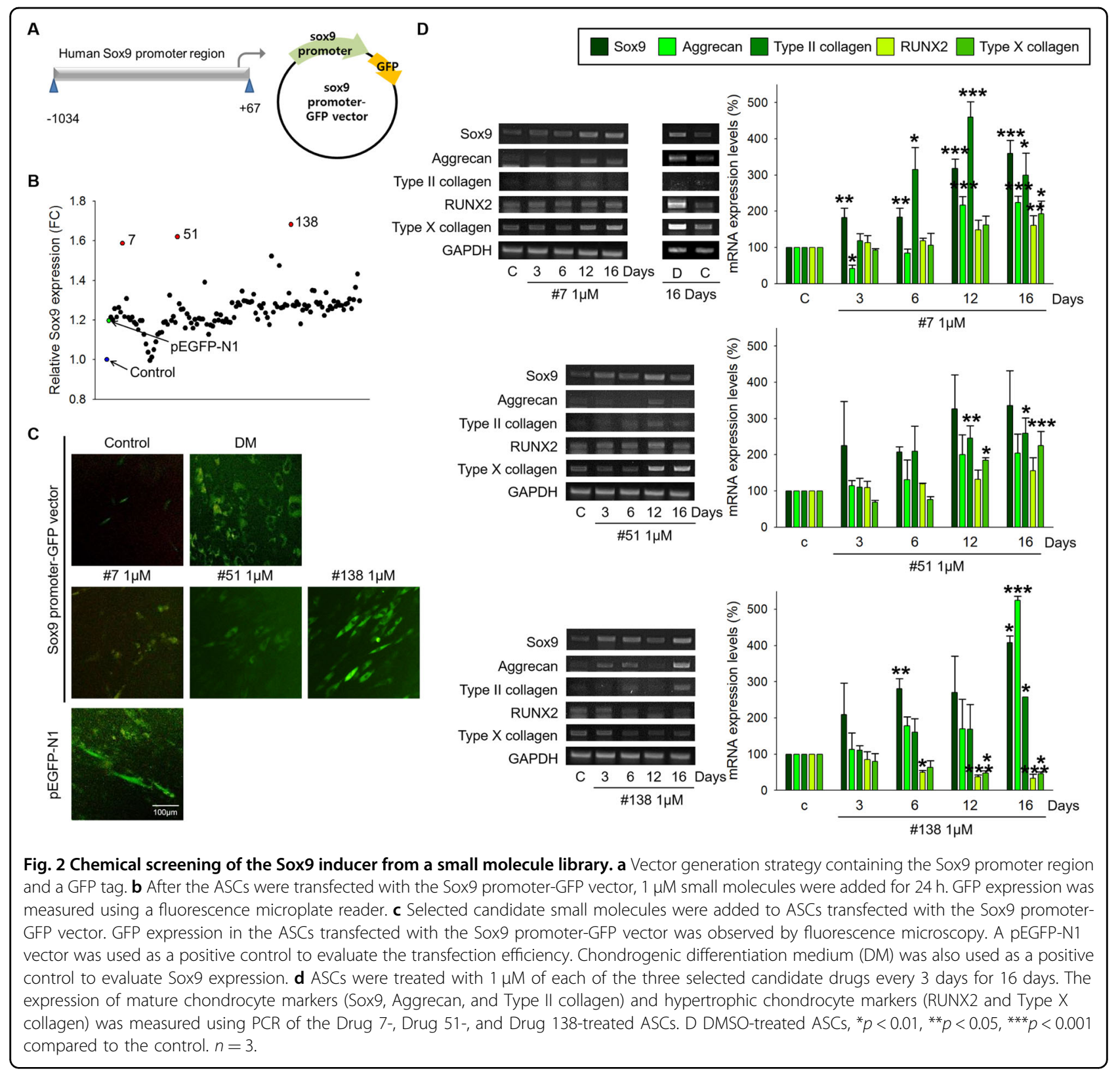

formaldehyde solution for $1 \mathrm{~h}$ at room temperature. Then, Alcian blue staining solution with $3 \%$ acetic acid solution $(\mathrm{pH} 2.5)$ was added to each sample for $30 \mathrm{~min}$. After staining, the samples were washed in running tap water for $2 \mathrm{~min}$ and finally mounted with mounting solution.

\section{Safranin $\mathrm{O}$ staining}

The knee joint paraffin sections were stained using a safranin $O$ staining kit. First, the section was deparaffinized, rehydrated, rinsed with tap water and then stained with $0.1 \%$ fast green solution for $5 \mathrm{~min}$. The sample was rinsed with $1 \%$ acetic acid for $10 \mathrm{~s}$ and then stained using $0.1 \%$ safranin $O$ staining solution for $30 \mathrm{~min}$.
Finally, the section was dehydrated and mounted with mounting solution.

\section{Immunofluorescence}

The knee joint was fixed overnight with $10 \%(\mathrm{v} / \mathrm{v})$ formaldehyde. The knee joint was embedded in paraffin and transversely sectioned into serial thick sections. After deparaffinization, rehydration, and rinsing with tap water, sodium citrate antigen retrieval was performed using $10 \mathrm{mM}$ sodium citrate ( $\mathrm{pH} \mathrm{6.0)}$ in a microwave for $10 \mathrm{~min}$. Sections were incubated in $1 \% \mathrm{H}_{2} \mathrm{O}_{2}$ to quench endogenous peroxidase. The tissue sections were treated with $0.1 \%$ sodium borohydride to remove autofluorescence and 


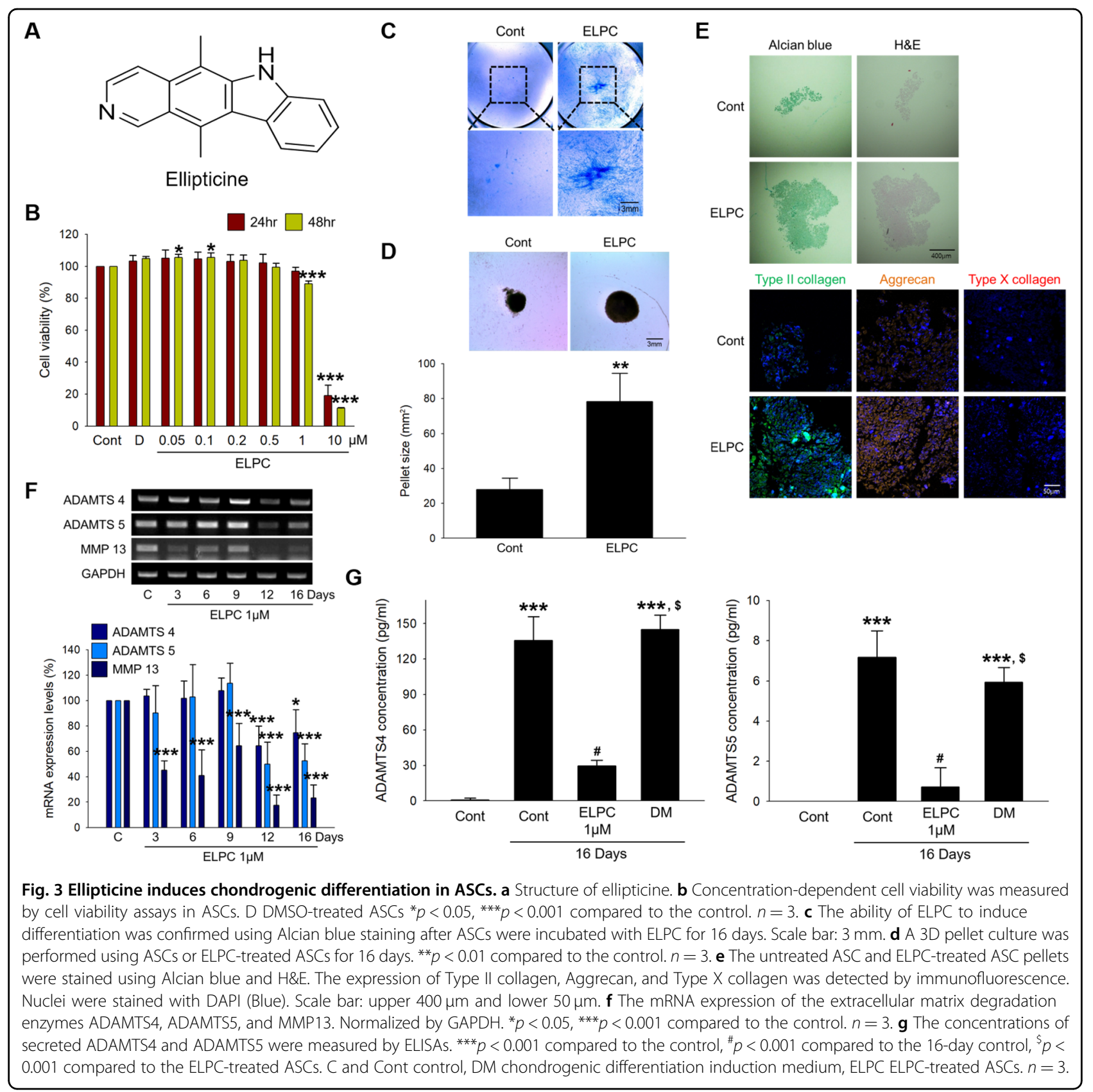

were blocked in 2.5\% normal horse serum. After blocking, the sections were incubated with primary antibody overnight. The sections were analyzed for DAPI, Aggrecan, and collagen type 2 and 10 by an LSM700 confocal laser scanning microscope.

\section{Statistical analysis}

The results are expressed as the mean \pm SD from at least three independent experiments. Statistical analyses were performed using Student's $t$-test. Comparisons between more than two groups were performed by one-way ANOVA using Bonferroni's correction. Relationships were considered statistically significant when the $p$-value was $<0.05$.

\section{Results}

Screening of small molecules that induce the differentiation of ASCs into chondrocytes

First, we found that the mRNA and protein levels of the sex-determining region Y-type high mobility group box (Sox) 9 were higher in chondrocytes than in ASCs (Fig. 1a, b). Next, we confirmed an important role of Sox9 during the differentiation of ASCs into chondrocytes. The increased expression of Sox9, Aggrecan, and Type II 


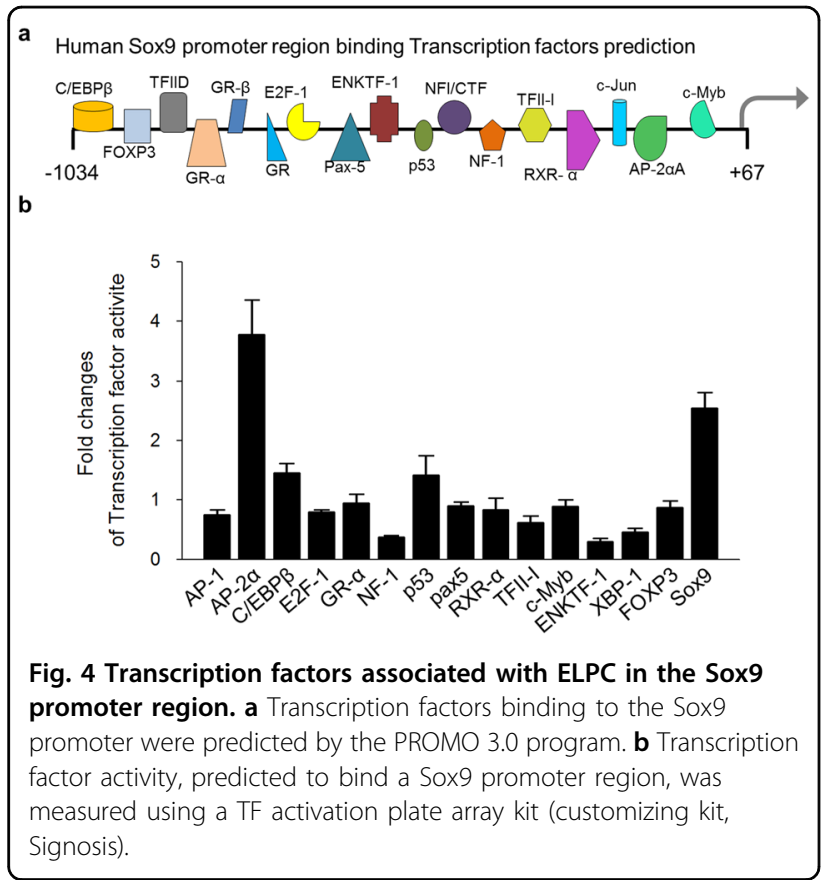

collagen in the DM-treated ASCs was inhibited in the Sox9 siRNA-transfected ASCs (Fig. 1c). A specific vector system containing the Sox 9 promoter region and GFP was used to screen for a drug that increases Sox9 expression (Fig. 2a). The relative Sox9 expression was analyzed based on the overexpression of GFP under the treatment of small molecules (Fig. 2b) and was confirmed using fluorescence microscopy (Fig. 2c). Finally, three candidate drugs were selected, and the differentiation capability of the drugs was determined by the mRNA expression levels of mature chondrocyte and hypertrophic chondrocyte markers. The expression levels of Sox9, Aggrecan, and Type II collagen were increased in the ASCs treated with each of the three drugs, whereas those of RUNX2 and Type X collagen were increased in the cells treated with drugs 7 and 51 and not in the ASCs treated with drug 138, as shown in Fig. 2d.

\section{ELPC induces chondrocyte differentiation in adipose- derived stem cells}

Drug 138, known as ellipticine (ELPC), is a natural tetracyclic compound (Fig. 3a). The cytotoxicity in ASCs prior to further experiments was investigated; $1 \mu \mathrm{M}$ ELPC resulted in a slight reduction in cell viability, and $80 \%$ or more of the ASCs underwent cell death with $10 \mu \mathrm{M}$ ELPC (Fig. 3b). To further confirm the ability of ELPC to induce the differentiation of ASCs into chondrocytes, we performed Alcian blue staining after the ASCs were incubated with ELPC for 16 days. Figure 3c shows that the ELPCtreated ASCs had more blue and condensed areas than the untreated ASCs. The ELPC-treated ASCs maintained more rounded pellets and a greater diameter and total size than the untreated ASCs (Fig. 3d). The expression levels of Aggrecan and Type II collagen were increased in the ELPC-treated ASC pellets, whereas the expression of Type $\mathrm{X}$ collagen was not increased (Fig. 3e). We also confirmed that the mRNA levels of ECM-degrading enzymes were decreased in a time-dependent manner by ELPC treatment (Fig. 3f). In addition, the levels of ADAMTS4 and ADAMTS5 were significantly decreased in the medium of the ELPC-treated ASCs (Fig. 3g).

ELPC increases the expression of Sox 9 by regulating $\mathrm{p} 53$ during chondrogenesis

We investigated whether ELPC regulates transcription factors that can bind to the promoter region of Sox9. Several transcription factors were selected that were predicted to bind to the Sox9 promoter region by the PROMO 3.0 program. Among them, p53 was found to be associated with ELPC (Fig. 4). Therefore, the mRNA and protein levels of p53 were measured after ELPC treatment. Both the mRNA and protein levels of p53 increased for up to $24 \mathrm{~h}$ (Fig. 5a, b). The expression of p53 was also evaluated in isolated nuclear and cytosolic fractions. p53 expression was shifted to the nucleus (Fig. 5c), and a fluorescent stain of p53 also showed translocation from the cytoplasm to the nucleus following ELPC treatment (Fig. 5d). We further confirmed the association between ELPC and p53. The expression of p53 was decreased by p53 siRNA (Fig. S1), which inhibited the expression of Sox 9 and Type II collagen (Fig. 5e, f). In addition to p53, we also investigated whether AP- $2 \alpha$ or C/EBP $\beta$ siRNA regulated the expression of Sox9, resulting in no reduction in mature chondrocyte markers (data not shown). These results indicate that ELPC induces Sox 9 expression by regulating the expression of p 53 during the chondrogenesis of ASCs.

\section{Protective effects of ELPC in an animal model of osteoarthritis}

A cartilage-protective effect of ELPC-treated ASCs was observed in an animal model of OA. Decreased cartilage damage was observed in the ASC- and ELPC-treated ASC-injected groups (Fig. 6a). The expression levels of both Type II collagen and Aggrecan were higher than those of the OA-induced group, while the expression level of Type X collagen was lower in the ELPC-treated ASCinjected group than in the ASC-injected group (Fig. 6b).

\section{Discussion}

Our results suggest that ELPC can induce ASC differentiation into chondrocytes through upregulation of the expression of Sox9 by increasing the expression and activation of p53. Most importantly, ASCs differentiated by ELPC did not exhibit the characteristics of hypertrophic chondrocytes. In these experiments, we used the 


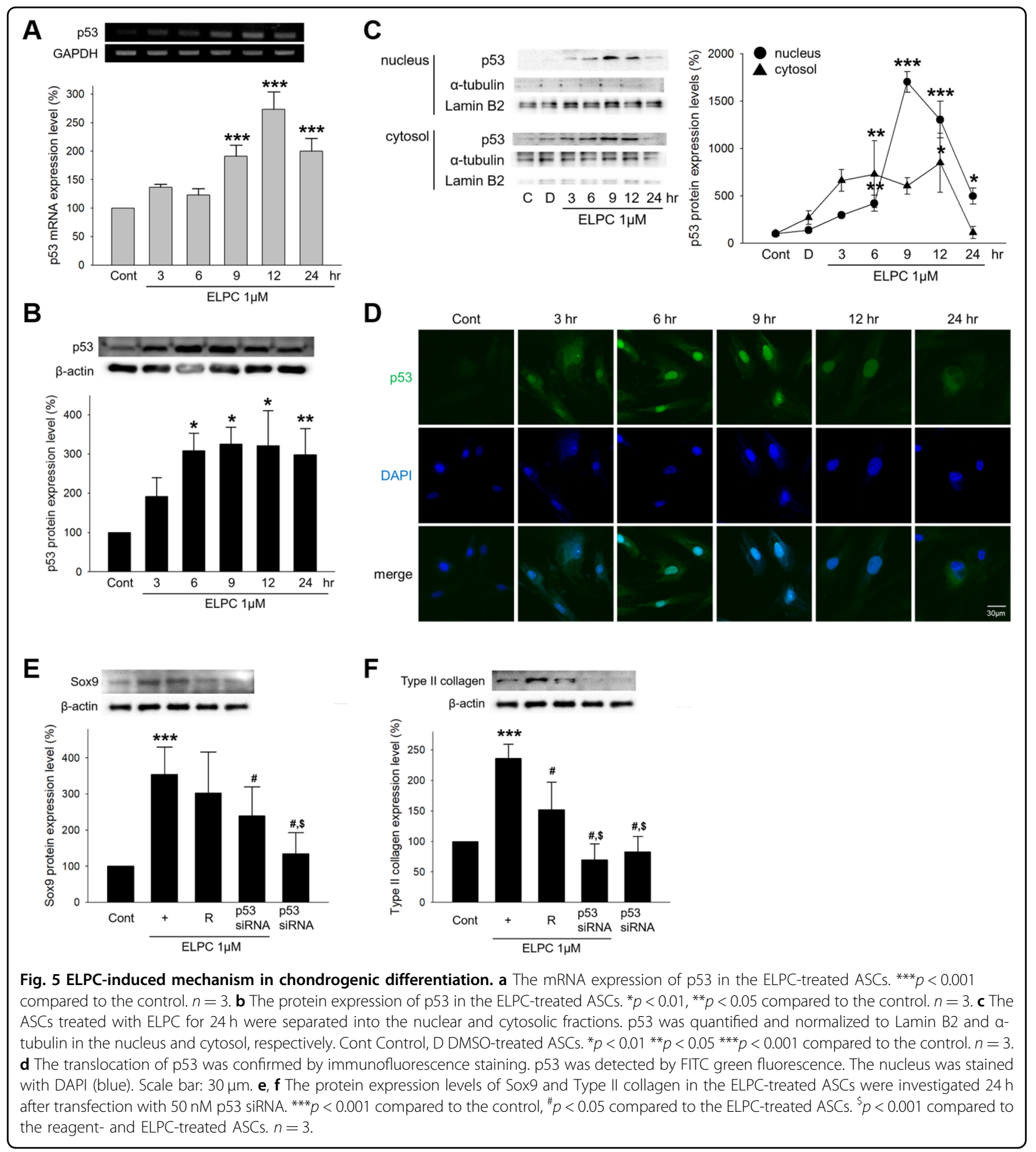

Sox9 promoter-GFP vector system to screen small molecules because Sox9 has a critical role as a transcription factor during cartilaginous skeleton formation in the early stage of chondrogenesis ${ }^{21-23}$ and regulates chondrocyte-specific matrix proteins such as Type II collagen and Aggrecan ${ }^{24,25}$. Therefore, we finally chose ELPC, an alkaloid that was first extracted from the species
Ochrosia elliptica and Rauvolfia sandwicensis. In cancer, ELPC has been used as a drug to induce cell death because it inhibits the enzyme topoisomerase II via intercalative binding to $\mathrm{DNA}^{26}$. Therefore, we assessed cell viability and found there was no toxicity until $0.5 \mu \mathrm{M}$. In this study, Sox 9 overexpression by ELPC treatment was critical, and p53 was shown to play an important role in 
A
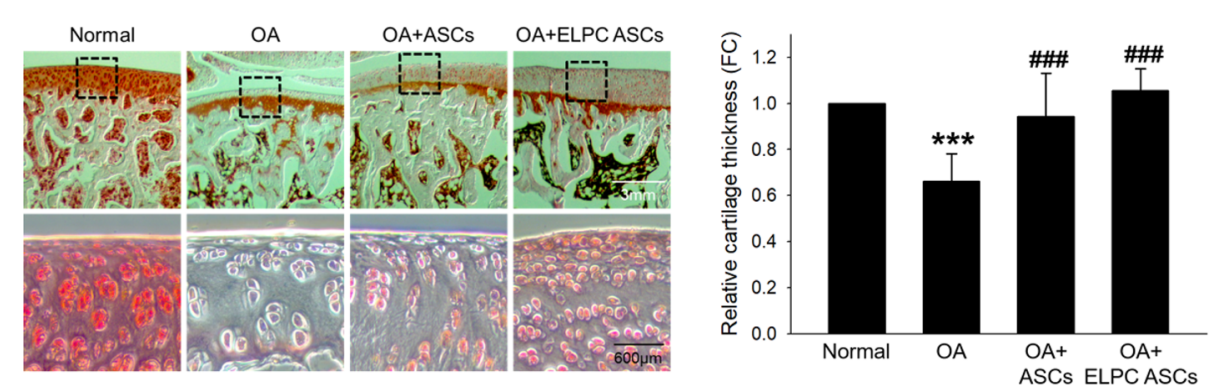

B

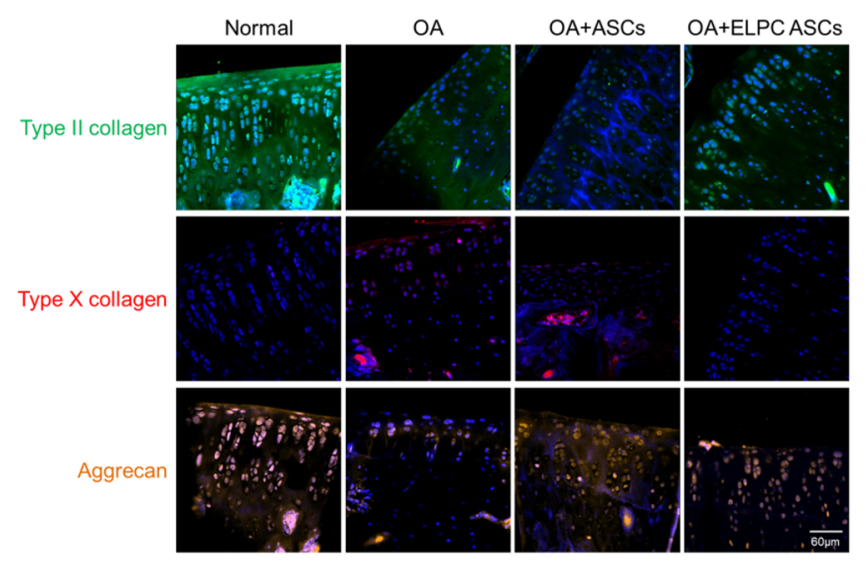

Fig. 6 ELPC-induced mechanism and protective effect in an animal model of OA. a Safranin O staining of the paraffin sections of the OA animal model. Representative images show cartilage damage after 6 weeks of OA induction. Safranin O binds to glycosaminoglycan in cartilage and is shown in red. The lower figure shows an enlargement of the cartilage part in the upper figure, scale bar: upper $3 \mathrm{~mm}$ and lower $600 \mu \mathrm{m}$. Graph indicating the relative cartilage thickness. ${ }^{* *} p<0.001$ compared to the normal control, ${ }^{\# \#} p<0.001$ compared to the animal model of OA. $n=3$. b Detection of mature and hypertrophic chondrocyte markers in the OA animal model. Type II collagen (FITC, green), aggrecan (Texas red, red), and type $X$ collagen (rhodamine, orange) expression was measured by immunofluorescence staining and visualized with a confocal microscope. Each section was costained with DAPI (blue) for nuclear staining. The images in the same region of tissue were merged into the overlapped image. Scale bar: $60 \mu \mathrm{m}$.

regulating Sox9 expression. Consequently, ELPC increased the expression and nuclear translocation of p53. Most previous studies of ELPC have explored its effects on various cancers. ELPC inhibited p53 ubiquitination and increased the nuclear localization of endogenous $\mathrm{p} 53^{4,27}$. Another study also demonstrated that ELPC recovered the transcriptional function of mutant $\mathrm{p} 53^{27}$. Recent studies have confirmed that p53 regulates the differentiation of embryonic stem cells and induces pluripotent stem cells in response to genomic damage, similar to our results ${ }^{28,29}$.

In vivo studies demonstrated that cartilage damage could be recovered in rats injected with ASCs differentiated by ELPC. In addition, the indicators of mature chondrocytes were increased, and indicators of hypertrophic chondrocytes were not increased (Fig. 6). The limitations of this study include a lack of confirmation of whether differentiated ASCs directly adhere to cartilage to regenerate or are affected by secreted molecules from transplanted ASCs. Therefore, further investigation is needed to confirm these issues. In addition, since there are stem cells in the synovium, it is also necessary to confirm the effect of ELPC treatment alone in amelioration of $\mathrm{OA}$ in an in vivo study.

Taken together, the results demonstrate that ELPC induces differentiation into the appropriate type of chondrocytes by increasing Sox9 from ASCs, and ELPC exerted a significant protective effect in an animal model of OA. These results suggest that ELPC is a potential new therapeutic drug for OA.

\section{Acknowledgements}

This research was supported by grants from the Ministry of Health \& Welfare, Republic of Korea (H18C0661), and the Basic Science Research Program through the National Research Foundation of Korea (NRF) funded by the Ministry of Education (2018R1A6A3A01012013).

\section{Author details}

${ }^{1}$ Brain Korea 21 PLUS Project for Medical Science, Yonsei University, Seoul, Republic of Korea. ${ }^{2}$ Institute for Bio-Medical Convergence, College of Medicine, Catholic Kwandong University, Gangneung, Republic of Korea. ${ }^{3}$ Department of Integrated Omics for Biomedical Sciences, Yonsei University, Seoul, Republic of Korea. ${ }^{4}$ Department of Medical Engineering, Yonsei University College of Medicine, Seoul 03722, Republic of Korea 


\section{Conflict of interest}

The authors declare that they have no conflict of interest.

\section{Publisher's note}

Springer Nature remains neutral with regard to jurisdictional claims in published maps and institutional affiliations.

Supplementary information accompanies this paper at https://doi.org/ 10.1038/s12276-020-0424-y.

Received: 1 October 2019 Revised: 17 January 2020 Accepted: 10 February 2020.

Published online: 21 April 2020

\section{References}

1. Loeser, R. F. Age-related changes in the musculoskeletal system and the development of osteoarthritis. Clin. Geriatr. Med. 26, 371-386 (2010).

2. Arden, N. \& Nevitt, M. C. Osteoarthritis: epidemiology. Best. Pract. Res. Clin. Rheumatol. 20, 3-25 (2006).

3. Grotle, M., Hagen, K. B., Natvig, B., Dahl, F. A. \& Kvien, T. K. Obesity and osteoarthritis in knee, hip and/or hand: an epidemiological study in the general population with 10 years follow-up. BMC Musculoskelet. Disord. 9, 132 (2008).

4. Spector, T. D. \& MacGregor, A. J. Risk factors for osteoarthritis: genetics. Osteoarthr. Cartil. 12, S39-44 (2004).

5. Ettinger, W. H. Jr et al. A randomized trial comparing aerobic exercise and resistance exercise with a health education program in older adults with knee osteoarthritis. The Fitness Arthritis and Seniors Trial (FAST). JAMA 277, 25-31 (1997).

6. Sophia Fox, A. J., Bedi, A. \& Rodeo, S. A. The basic science of articular cartilage: structure, composition, and function. Sports Health 1, 461-468 (2009).

7. Kuete, V. \& Efferth, T. Cameroonian medicinal plants: pharmacology and derived natural products. Front. Pharmacol. 1, 123 (2010).

8. D'Angelo, M. et al. MMP-13 is induced during chondrocyte hypertrophy. J. Cell. Biochem. 77, 678-693 (2000).

9. Yamamoto, K. et al. MMP-13 is constitutively produced in human chondrocytes and co-endocytosed with ADAMTS-5 and TIMP-3 by the endocytic receptor LRP1. Matrix biology: journal of the International Society for. Matrix Biol. 56, 57-73 (2016).

10. Coggon, D. et al. Knee osteoarthritis and obesity. Int. J. Obes. Relat. Metab. Disord. 25, 622-627 (2001)

11. Bunnell, B. A., Flaat, M., Gagliardi, C., Patel, B. \& Ripoll, C. Adipose-derived stem cells: isolation, expansion and differentiation. Methods 45, 115-120 (2008).

12. Becker, A. J., McCulloch, E. A. \& Till, J. E. Pillars article: cytological demonstration of the clonal nature of spleen colonies derived from transplanted mouse marrow cells. Nature. 1963. 197:452-454. J. Immunol. 192, 4945-4947 (2014).
13. Oseni, A. O., Butler, P. E. \& Seifalian, A. M. Optimization of chondrocyte isolation and characterization for large-scale cartilage tissue engineering. J. Surg. Res. 181, 41-48 (2013)

14. Goldring, M. B., Tsuchimochi, K. \& ljiri, K. The control of chondrogenesis. J. Cell. Biochem. 97, 33-44 (2006).

15. Rupaimoole, R., Han, H. D., Lopez-Berestein, G. \& Sood, A. K. MicroRNA therapeutics: principles, expectations, and challenges. Chin. J. Cancer 30, 368-370 (2011).

16. Krichevsky, A. M., Sonntag, K. C., Isacson, O. \& Kosik, K. S. Specific microRNAs modulate embryonic stem cell-derived neurogenesis. Stem Cells $\mathbf{2 4}, 857-864$ (2006).

17. Yang, B. et al. MicroRNA-145 regulates chondrogenic differentiation of mesenchymal stem cells by targeting Sox9. PloS one 6, e21679 (2011).

18. Hwang, K. C. et al. Chemicals that modulate stem cell differentiation. Proc. Nat/ Acad. Sci. USA 105, 7467-7471 (2008)

19. Colter, D. C. et al. Regulation of the human Sox9 promoter by the CCAATbinding factor. Matrix biology: journal of the International Society for. Matrix Biol. 24, 185-197 (2005).

20. Johnstone, B., Hering, T. M., Caplan, A. I., Goldberg, V. M. \& Yoo, J. U. In vitro chondrogenesis of bone marrow-derived mesenchymal progenitor cells. Exp. Cell Res. 238, 265-272 (1998).

21. Jo, A. et al. The versatile functions of Sox9 in development, stem cells, and human diseases. Genes Dis. 1, 149-161 (2014).

22. Ikeda, T. et al. The combination of SOX5, SOX6, and SOX9 (the SOX trio) provides signals sufficient for induction of permanent cartilage. Arthritis Rheum. 50, 3561-3573 (2004).

23. Bell, K. M., Western, P. S. \& Sinclair, A. H. SOX8 expression during chick embryogenesis. Mech. Dev. 94, 257-260 (2000).

24. Lefebvre, V., Huang, W., Harley, V. R., Goodfellow, P. N. \& de Crombrugghe, B. SOX9 is a potent activator of the chondrocyte-specific enhancer of the pro alpha1(II) collagen gene. Mol. Cell. Biol. 17, 2336-2346 (1997).

25. Akiyama, H., Chaboissier, M. C., Martin, J. F., Schedl, A. \& de Crombrugghe, B. The transcription factor Sox9 has essential roles in successive steps of the chondrocyte differentiation pathway and is required for expression of Sox5 and Sox6 Gene Dev. 16, 2813-2828 (2002).

26. Auclair, C. Multimodal action of antitumor agents on DNA: the ellipticine series. Arch. Biochem. Biophys. 259, 1-14 (1987).

27. $\mathrm{Xu}, \mathrm{G} . \mathrm{W}$. et al. A high-content chemical screen identifies ellipticine as a modulator of p53 nuclear localization. Apoptosis 13, 413-422 (2008).

28. Li, M. et al. Distinct regulatory mechanisms and functions for p53-activated and p53-repressed DNA damage response genes in embryonic stem cells. Mol. Cell 46, 30-42 (2012).

29. Lin, T. \& Lin, Y. p53 switches off pluripotency on differentiation. Stem Cell Res. Ther. 8, 44 (2017). 